\title{
Diabetes Mellitus tipo 2: Aspectos fisiológicos, genéticos e formas de exercício físico para seu controle
}

\author{
Type 2 Diabetes Mellitus: Physiological and genetic aspects \\ and the use of physical exercise for diabetes control
}

\author{
Gisela Arsa ${ }^{1,3}$ \\ Laila Lima ${ }^{1,3}$ \\ Sandro Soares de Almeida ${ }^{2}$ \\ Sérgio Rodrigues Moreira ${ }^{1}$ \\ Carmen Sílvia Grubert Campbell ${ }^{1}$ \\ Herbert Gustavo Simões
}

1 Universidade Católica de Brasília. Programa de Mestrado e Doutorado em Educação Física. DF. Brasil

2 Universidade de Mogi das Cruzes. Programa de Mestrado e Doutorado em Biotecnologia. SP. Brasil.

3 Bolsista CAPES.

Recebido em 14/12/07 Aprovado em 16/06/08
Resumo - Diabetes Mellitus tipo 2 (DM2) é uma doença metabólica caracterizada por hiperglicemia e disfunções cardiovasculares, as quais podem ser controladas com exercícios físicos, controle dietético e tratamento farmacológico. Pesquisas recentes têm demonstrado associações entre DM2 e alguns polimorfismos genéticos, em especial do gene da Enzima Conversora de Angiotensina (ECA). A proposta desse estudo foi abordar a fisiopatologia do DM2 e a prática de exercício físico como forma de controle não farmacológico da glicemia e pressão arterial destes pacientes, bem como a associação entre o DM2 e polimorfismos do gene da ECA. A pesquisa incluiu livros da área de saúde, bem como artigos de revisão e originais referentes ao tema abordado, pesquisados nos bancos de dados disponíveis na internet: Pubmed, Scielo e Portal Capes. As palavras chave utilizadas foram "blood glucose", "type 2 diabetes", "exercise", "blood pressure" e "ACE gene polymorphism", e suas traduções para a língua portuguesa. Os principais assuntos do presente artigo referem-se à prática diária de exercícios, tipo e intensidades adequadas que têm se mostrado eficazes no controle glicêmico e hemodinâmico, assim como as possíveis relações com os polimorfismos da ECA, que ainda se apresentam incipientes, pois a constatação de associações entre estes podem ser fortemente influenciadas pelo tipo de população estudada. Os benefícios do exercício são indiscutíveis e suas recomendações apresentadas nesta revisão. Porém a literatura ainda é carente de estudos analisando os efeitos do exercício físico para diabéticos, considerando sua relação com aspectos genéticos, o que coloca este assunto em evidência como objeto de estudo na atualidade.

Palavras-chave: Diabetes Mellitus; Exercício físico; Polimorfismo genético.

Abstract - Type 2 Diabetes mellitus (DM2) is a metabolic disease characterized by hyperglycemia and cardiovascular dysfunctions that can be controlled with physical exercise, dietary control and pharmacological treatment. Recent research has demonstrated associations between DM2 and some genetic polymorphisms, especially alterations to the gene that codes for Angiotensin Converting Enzyme (ACE). The purpose of this study was to discuss the pathophysiology of DM2 and the use of physical exercise as a non-pharmacological method for controlling these patients' glycemia and blood pressure. The relationship between DM2 and polymorphisms of the ACE gene was also covered. The literature search included textbooks in the healthcare field in addition to review articles and original articles found on the databases Pubmed, Scielo and Portal Capes. Keywords used were "blood glucose", "type 2 diabetes", "exercise", "blood pressure" and "ACE gene polymorphism" and their translations in Portuguese. The main subject of this article is the practice of daily exercise, the types and intensities that have proven effective for glycemic and haemodinamic control and possible relationships with the ACE polymorphism, which are currently still tentative, since the evidence of associations can be strongly influenced by the population studied. The benefits of exercise are unquestionable and the recommended forms are discussed in this review. However the literature is still lacking studies that analyze the effects of physical exercise on diabetics and take into account the relationship with genetic aspects. This area is evidently an appropriate subject for further research.

Key words: Diabetes mellitus; Physical exercise: Genetic polymorphisms. 


\section{INTRODUÇÃO}

O Diabetes Mellitus (DM) é uma doença endócrina caracterizada por um grupo de desordens metabólicas, incluindo elevada glicemia de jejum (hiperglicemia) e elevação das concentrações de glicose sanguínea pós-prandial, devido a uma menor sensibilidade insulínica em seus tecidos alvo e/ou por reduzida secreção de insulina ${ }^{1}$.

De acordo com a $\mathrm{ADA}^{1}$, existem 4 classificações de DM: tipo 1 ou insulino-dependente (DM1); tipo 2 ou não insulino-dependente (DM2); gestacional; e secundário a outras patologias. Independente da classificação, a principal característica do DM é a manutenção da glicemia em níveis acima dos valores considerados normais. O retardo para o início do tratamento do DM pode acarretar no desenvolvimento de doenças cardiovasculares, retinopatias, neuropatias autonômicas e periféricas, nefropatias, doença vascular periférica, aterosclerose, doença cerebrovascular, hipertensão, susceptibilidade a infecções e doenças peridontais.

O DM tipo 2 é associado a fenótipos como o sedentarismo e a obesidade, e esses fenótipos interagem com alguns genes que podem ser responsáveis por uma maior susceptibilidade a essa patologia ${ }^{2}$.

O gene da ECA (Enzima Conversora de Angiotensina) tem sido considerado um candidato que pode estar envolvido nessas respostas metabólicas diferenciadas entre os indivíduos. Seus polimorfismos apresentados são de deleção (alelo D) ou inserção (alelo I), que resultam em alta ou baixa atividade da ECA, respectivamente, a qual atua no sistema renina-angiotensina, convertendo a angiotensina I (Ang I) em angiotensina II (Ang II), um potente vasoconstritor, causando elevação da pressão arterial $^{3}$.

Níveis aumentados de ECA circulantes, observados na presença do alelo $\mathrm{D}$, resultam em elevação da pressão arterial quando comparado a indivíduos que apresentam o alelo $\mathrm{I}^{4}$, e também resultam em menor biodisponibilidade da enzima bradicinina (BK), responsável por promover vasodilatação ${ }^{5}$ e diminuição da resistência a insulina nas células musculares ${ }^{6}$.

Diabéticos tipo 2 apresentam 1,77 mais vezes o polimorfismo de deleção nos dois alelos homozigotos DD - quando comparado a pessoas saudáveis (48\% em diabéticos e 27\% em não diabéticos) $)^{7}$, o que pode resultar em respostas diferenciadas ao exercício físico, considerando que $52 \%$ desses diabéticos podem ser homozigotos para inserção (II) ou heterozigotos (ID).

Ainda assim, o controle do DM2 pode ser feito por meio da associação de uma dieta alimentar adequada de baixo índice glicêmico, prática de exercício físico e uso de medicamentos hipoglicemiantes ${ }^{1}$.

Muitos estudos ${ }^{8-11}$ têm demonstrado que o exercício físico resulta em melhoras significativas para os portadores de DM2, como a redução da glicemia após a realização de exercício, redução da glicemia de jejum, da hemoglobina glicada (HbA1c), bem como melhora da função vascular ${ }^{2}$.

Os estudos ainda não esclarecem totalmente as associações observadas entre o polimorfismo do gene da ECA, diabetes tipo 2, sistema renina-angiotensina e exercício físico. Mediante isso, essa revisão de literatura tem como objetivo abranger aspectos relacionados a fisiopatologia da doença em questão, incluindo associações genéticas do polimorfismo da ECA a fim de colaborar para o maior entendimento dos danos que o DM2 ocasiona, o papel do exercício físico como tratamento não medicamentoso do diabetes tipo 2 e, finalmente, abordar as possíveis relações entre as respostas ao exercício e polimorfismos do gene da ECA.

\section{DIABETES MELLITUS E PREVALÊNCIA}

Pessoas adultas acima dos 40 anos são mais acometidas por DM2, que pode vir acompanhado de obesidade, hipertensão arterial sistêmica (HAS), dislipidemia, e disfunção endotelial. Sintomas como sede e diurese excessiva, dores nas pernas, alterações visuais e aumento de peso ${ }^{1}$ são característicos de portadores de DM2.

O DM2 é caracterizado pela incapacidade da insulina exercer normalmente suas funções. A resistência à ação da insulina impede o desencadeamento de respostas enzimáticas, que envolvem a auto-fosforilação da tirosina-quinase para o substrato IRS-1 ${ }^{12}$ e IRS-2 (substrato do receptor de insulina 1 e 2), os quais fosforilam diversas proteínas, como o fosfatidilinositol 3 quinase (PI 3-quinase), que está associada à síntese e translocação dos transportadores de glicose (GLUT) para a membrana celular. Assim, no DM2, a translocação de GLUT-4 
(transportador de glicose 4) e a captação de glicose pelas células ficam prejudicadas, levando à hiperglicemia crônica ${ }^{13}$.

Além da hiperglicemia crônica, anormalidades no metabolismo de lipídios (excesso de ácidos graxos livres circulantes no sistema portal) são observadas com freqüência em diabéticos tipo $2^{1}$, contribuindo para a formação de ateromas e aparecimento de lesões no músculo liso dos vasos sanguíneos, além de disfunções endoteliais desencadeadas pela resistência à insulina ${ }^{14}$.

O DM é uma doença de ordem mundial, que freqüentemente acomete adultos, sendo que para o ano 2000 a estimativa foi de 171 milhões de novos casos. Calcula-se que esse número alcance, aproximadamente, 366 milhões até 2030. A população urbana, mais afetada por essa patologia, deve dobrar em países em desenvolvimento entre 2000 e 2030. Esta mudança demográfica está associada ao aumento na prevalência de diabetes, além do aumento da proporção de pessoas com mais de 65 anos de idade, mais freqüentemente acometidas por essa doença. No Brasil, acredita-se que esse número alcance 11,3 milhões de pessoas ${ }^{15}$.

Além disso, o DM2 aumenta em 2 a 3 vezes o risco de se desenvolver doença arterial coronariana, e a maior incidência de mortalidade em diabéticos tipo 2 está intimamente relacionada ao estado diabético e à associação da doença com outros fatores de risco cardiovasculares, como sedentarismo, obesidade, hipertensão arterial sistêmica, dislipidemia, entre outros ${ }^{16}$.

\section{INSULINA E DIABETES TIPO 2}

A insulina é um hormônio importante para a manutenção da homeostase glicêmica e também para o crescimento e diferenciação celular. Tem função anabólica e é secretada pelo pâncreas (células $\beta$ das Ilhotas de Langerhans), em função da elevação da glicemia, dos níveis circulantes de aminoácidos e de ácidos graxos livres, como ocorre após a realização de refeições. Esta secreção aumentada de insulina promove a captação de glicose pelas células adiposas e musculares, inibindo processos catabólicos ${ }^{17}$.

A captação de glicose é possível devido a inúmeras reações enzimáticas desencadeadas pela insulina quando em contato com seu receptor transmembrana, sendo uma subunidade $\alpha$, localizada no exterior da célula, e uma su- bunidade $\beta$, localizada no citoplasma da célula. A subunidade $\alpha$ possui o sítio de ligação para a insulina e a subunidade $\beta$ transmite os sinais e possui atividade tirosina quinase, com característica de autofosforilação, fosforilando a família IRS, que então ativa a PI 3-quinase e esta por sua vez ativa a translocação do GLUT para a superfície da membrana celular, possibilitando a captação de glicose ${ }^{18}$.

A inibição da ação da insulina leva ao desenvolvimento de resistência à insulina e do DM2, podendo ser ocasionada pela adiposidade central, fator clássico para o aparecimento da resistência à insulina, estando associada ao aumento dos níveis de triglicérides, baixas concentrações de lipoproteína de alta densidade (HDL) e pressão arterial aumentada ${ }^{18}$ resultando no desenvolvimento de disfunções macrovasculares.

Essa resistência à insulina pode ser resultado de alterações intracelulares, nos pós-receptores de insulina, na interação IRS e PI 3-quinase, diminuindo a translocação dos transportadores de glicose para a superfície da membrana, alterações relacionadas com fatores como o excesso de ácidos graxos livres circulantes, inflamação e estresse oxidativo do retículo endoplasmático, aumentando a ativação de algumas enzimas que resultam em inativação do IRS ${ }^{19}$.

A resistência à insulina, com inibição do IRS-1, leva a uma hiperinsulinemia compensatória e ao desenvolvimento de disfunção endotelial, uma vez que o IRS-1 inibido impede a ativação da PI 3-quinase, que está envolvida na geração do estímulo para a produção de óxido nítrico nas células endoteliais. Com a diminuição do estímulo para a produção do óxido nítrico, a atividade contrátil da angiotensina II se torna mais evidente, produzindo vasoconstrição e redução da contratilidade vascular ${ }^{14}$, bem como nefropatia, retinopatia, neuropatia e hipertensão ${ }^{19}$.

\section{FATORES GENÉTICOS}

Polimorfismos de Inserção e Deleção do Gene da ECA: Relações com o Diabetes Mellitus Tipo 2 e a Hipertensão Arterial Sistêmica

O Sistema Renina-Angiotensina (SRA) é responsável pela homeostase de sódio e pela regulação da pressão arterial, tendo a Ang II como 
o principal efetor desse sistema. A formação da Ang II é dada pela ação da ECA, responsável pela conversão da Ang I, a qual tem pouco ou nenhum efeito sobre a pressão arterial, em Ang II, um potente vasoconstritor, atuando sobre os receptores AT1 e AT2 ${ }^{20}$.

A ECA tem presença abundante na superfície endotelial do pulmão e além de formar Ang II, também inativa a ação de peptídeos vasoativos como a bradicinina (BK) e a calidina ${ }^{4}$.

Polimorfismos de Inserção (I) e Deleção (D) para o gene da ECA, responsável pela expressão dessa enzima, podem estar associados com níveis normais ou elevados da ECA circulante. Os polimorfismos podem ser do tipo ID (heterozigotos inserção/deleção), II (homozigotos para inserção) e DD (homozigotos para deleção), estando este último associado a maiores concentrações e atividade da ECA ${ }^{3}$.

O gene da ECA está localizado no cromossomo 17 com inserção (alelo I) ou deleção (alelo D) de 287 pares de bases (pb) no intron $16^{2}$.

Arzu Ergen et al. ${ }^{7}$ verificaram polimorfismos do gene da ECA em diabéticos tipo 2 e não diabéticos, e a freqüência de polimorfismo de DD (homozigoto para deleção) em diabéticos tipo 2 foi de $48 \%$, enquanto que, em pessoas saudáveis, a presença desse polimorfismo DD foi de $27 \%$, estando o polimorfismo de DD do gene da ECA 1,77 vezes maior em diabéticos do que em pessoas saudáveis, sugerindo risco de desenvolvimento de nefropatia, já que o polimorfismo DD da ECA é considerado fator de risco independente para essa doença ${ }^{21}$.

Indivíduos que possuem o alelo D apresentam maior atividade da ECA circulante, a qual converte a Ang I em Ang II, promovendo vasocontrição. Como a ECA degrada a BK, responsável pela vasodilação, maiores níveis de ECA reduzem a biodisponibilidade de BK, potencializando a vasocontrição, e com isso níveis de pressão arterial mais elevados são observados em função do alelo D quando comparados a indivíduos que apresentam o alelo $\mathrm{I}^{4}$.

Níveis aumentados de ECA estão associados com a hipertensão arterial, hipertrofia cardía$\mathrm{ca}^{2}$, e com a nefropatia no DM2?

A BK também atua na captação de glicose pelas células musculares, ligando-se ao receptor de membrana B2, aumentando a ação da insulina, mas devido a sua menor biodisponibilidade pela ação da ECA na presença do alelo D, essa atuação é diminuída 6 .
Diabéticos tipo 2 homozigotos para deleção apresentam níveis maiores de insulina em jejum em relação a diabéticos homozigotos para inserção. Essas diferenças não foram verificadas em indivíduos não diabéticos ${ }^{22}$.

Molnar et al..$^{23}$ analisaram polimorfismos de I/D do gene da ECA em 145 diabéticos tipo 2 e verificaram que aqueles que carregam o alelo D foram os que apresentaram maiores níveis de frutosamina (maioria das proteínas glicosiladas circulantes) em relação aos que carregavam o alelo I. Além disso, os autores constataram um nível aumentado da atividade de gama-GT e de albuminúria, marcadores de estresse oxidativo e danos endoteliais respectivamente naqueles que carregam o alelo D. Assim, o alelo D pode contribuir para a elevação da glicação, bem como do estresse oxidativo, contribuindo com o desenvolvimento de alterações orgânicas prejudiciais em diabéticos tipo 2 .

O polimorfismo de I/D do gene da ECA foi associado com a existência de síndrome metabólica (SM) em Chineses com DM2, pois 86,1\% dos diabéticos tipo 2 tinham o genótipo DD de ECA e apresentavam SM, sugerindo que o sistema renina-angiotensina tem relação com danos metabólicos em diabéticos tipo $2^{24}$.

Costa et al..$^{25}$ pesquisaram polimorfismos de inserção/deleção do gene da ECA em brasileiros diabéticos, já que esse polimorfismo é considerado fator de risco para desencadear doenças como HAS, DM2 e doença arterial coronariana (DAC), porém não encontraram associação do polimorfismo com a presença de SM em pessoas diabéticas. Os pesquisadores atribuíram esses resultados a grande miscigenação dos brasileiros, sugerindo estudos que identifiquem a etnia prevalente no grupo pesquisado.

Já Wang et al. ${ }^{21}$ pesquisaram 1.281 Chineses com DM2 durante aproximadamente 41 meses, observando a ocorrência de problemas renais, cardiovasculares e mortes, analisando os polimorfismos de I/D do gene da ECA apresentados por esses pacientes. Foram constatadas associações do alelo D com o desenvolvimento de nefropatia na presença de outros fatores de risco como dislipidemia, hipertensão e DM2, devido a maior concentração da ECA circulante.

$\mathrm{O}$ uso de medicamentos inibidores da ação da ECA no organismo foi associado com diminuição nos riscos de mortalidade e ocorrência de falência renal em Chineses diabéticos tipo 2, mas esses benefícios foram mais evidentes entre 
diabéticos tipo 2 que carregam polimorfismo II e ID comparados aos diabéticos tipo 2 que apresentaram polimorfismo $\mathrm{DD}^{26}$.

Da mesma forma que os medicamentos podem resultar em maior ou menor atuação de acordo com o tipo de polimorfismo do gene da ECA em diabéticos tipo 2, talvez isso ocorra também com relação ao exercício físico. Essas interações genéticas e fisiológicas do DM2 não estão bem esclarecidas.

O que se sabe é que o exercício físico, quando realizado de forma adequada, promove benefícios importantes para o paciente diabético de forma mais ou menos aparente.

\section{Papel do Exercício Físico no controle glicêmico e hemodinâmico}

Os benefícios proporcionados pela prática de exercício físico no indivíduo diabético e que geralmente é hipertenso ${ }^{2}$ podem ser agudos (em curto prazo) ou crônicos (longo prazo).

Larsen et al. ${ }^{8}$ compararam o comportamento das concentrações sanguíneas de glicose e insulina pós-prandial em diabéticos tipo 2 após realização de exercício e/ou não (controle). Os pacientes submeteram-se a exercício físico em cicloergômetro, em intensidades intermitentes (3min a $57 \pm 4 \%$ do $\mathrm{VO}_{2 \max }, 4 \mathrm{~min}$ a $98 \pm 5 \%$ do $\mathrm{VO}_{2 \max }$ ), com intervalos de recuperação de 6 min, por $46 \mathrm{~min}$. Foram observados decréscimos consideráveis na glicemia e nas concentrações de insulina durante o exercício e também no período pós-exercício, quando comparados ao dia controle. Após 140 min de recuperação pós-exercício, os participantes receberam uma refeição, composta de carboidratos (53\%), lipídeos (31\%) e proteínas (16\%), sem diferenças significativas observadas para respostas da insulina e glicemia, entre as sessões de exercício e controle.

Poirier et al. ${ }^{9}$ avaliaram, em diabéticos tipo 2, as respostas da glicemia e insulinemia durante e após realização de exercício físico (1 hora a $60 \%$ do $\mathrm{VO}_{\text {2pico }}$ ) nas condições de jejum e alimentado. $\mathrm{O}$ efeito do exercício em reduzir a glicemia foi melhor quando os participantes se alimentaram duas horas antes de sua realização. Segundo os autores, a ingestão alimentar antes do exercício fez com que os praticantes realizassem o mesmo com a insulinemia mais elevada, e que somado ao efeito do exercício em captar glicose por vias independentes à ação da insulina, isto teria contribuído para um maior efeito hipoglicemiante pós-prandial em relação à condição jejum.

Alterações hormonais em resposta ao exercício resultam nesse efeito hipoglicemiante, via sinalização dos receptores de membrana, independentes da ação da insulina, a qual está inibida durante o exercício pela ação de catecolaminas (epinefrina e norepinefrina). Assim, o aumento da captação de glicose pelas células musculares, mediante a translocação do GLUT4 é feito por mecanismos independentes da ação insulínica, por meio de contrações musculares e/ou aumento da bradicinina plasmática ${ }^{5}$.

A utilização de fosfatos para produção de energia e o aumento da concentração de adenosina mono-fosfato (AMP), fosforilam e ativam as isoformas IRS-1 e IRS-2, associando-as a diversas proteínas, como a enzima fosfatidilinositol 3-quinase (PI 3-quinase), que provavelmente é responsável por ativar a translocação de GLUT4 no sarcolema, aumentando a captação de glicose durante o exercício ${ }^{13}$.

Taguchi et al. ${ }^{27}$, submeteram diabéticos tipo 2 a 20 minutos de exercício físico incremental em cicloergômetro e verificaram os níveis de bradicinina plasmática no repouso, a cada 5 minutos de exercício e ao término do exercício. Os diabéticos com elevados percentuais de HbAlc apresentavam menor formação de bradicinina após o exercício do que os diabéticos com percentuais menores de HbAlc.

A BK está envolvida na sinalização do GLUT-4, ativando sua translocação para a borda da membrana nas células musculares, aumentando a captação da glicose do sangue para o interior das células ${ }^{5}$, bem como melhorando a ação da insulina no período pós-exercício ${ }^{27}$.

Henriksen et al..$^{28}$, verificaram o aumento da BK por meio do decréscimo da ação da Ang II, inibindo a ECA em ratos obesos. Com a inibição da ECA houve maior estímulo para a captação de glicose no músculo. A maior disponibilidade de BK incrementou a ação da insulina, além de aumentar a produção de óxido nítrico.

A inibição da ECA pode reduzir a pressão arterial, bem como aumentar a ação da insulina na captação de glicose quando há o quadro de resistência à insulina ${ }^{28}$.

O exercício físico promove redução da pressão arterial abaixo dos valores observados no repouso, fenômeno denominado hipotensão pós-exercício (HPE). Esse fenômeno pode ser verificado em normotensos ${ }^{29}$ e hipertensos ${ }^{30} \mathrm{e}$ 
durar de $12^{3}$ a 22 horas $^{30}$ em hipertensos ${ }^{30}$, com decréscimo de até $20 \mathrm{mmHg}^{31}$.

MacDonald et al..$^{32}$ verificaram resposta hipotensora na pressão arterial média (PAM) em hipertensos "borderline", apresentando reduções significativas aos 5 e $15 \mathrm{~min}$ de recuperação pós-exercício, independente da intensidade de exercício (50 ou $75 \% \mathrm{VO}_{2 \text { pico }}$ ).

Brandão Rondon et al..$^{30}$ verificaram a HPE da pressão arterial sistólica e da PAM em hipertensos idosos, após exercício em cicloergômetro a $50 \%$ do $\mathrm{VO}_{2 \max }$, permanecendo diminuídas de forma significante quando comparadas com os valores obtidos no repouso, deixando em evidência o efeito hipotensor proporcionado pelo exercício de longa duração em hipertensos idosos.

Os mecanismos fisiológicos envolvidos na HPE estão associados, principalmente, à redução da resistência vascular periférica, mediada pela atuação do sistema de calicreínas-cininas, que promove vasodilatação por aumento da liberação da BK e conseqüentemente dilatação vascular $^{33}$, resultando em redução dos níveis pressóricos e contribuindo para redução de disfunções micro e macrovasculares.

A redução da pressão arterial após a realização de exercício foi verificada em hipertensos submetidos a corrida na água, devido ao aumento das concentrações de BK pós exercício ${ }^{34}$. O polimorfismo da ECA não foi identificado, bem como não foram avaliadas as concentrações de ECA. No entanto, talvez possa ter havido maior formação de BK e menor degradação da mesma por meio da ECA, devido à supressão do sistema renina-angiotensina.

Não se sabe ao certo se diabéticos tipo 2 apresentam essa mesma redução da pressão arterial pós-exercício, uma vez que dependendo dos valores de hemoglobina glicada a produção de BK pode ser maior ou menor pós-exercício ${ }^{27}$, além de também estarem associados ao polimorfismo da ECA.

Com relação aos benefícios crônicos obtidos pela prática de exercício físico, Silva e Lima ${ }^{10}$ submeteram diabéticos tipo 2 a um programa de 10 semanas de exercícios físicos aeróbios e resistidos combinados, e obtiveram reduções nos percentuais de $\mathrm{HbA1c}(-1,0 \%)$, reduções na glicemia de jejum $(-8,65$ mg.dL-1), freqüência cardíaca de repouso $(-10 \mathrm{bpm})$ e índice de massa corporal $\left(-0,65 \mathrm{~kg} / \mathrm{m}^{2}\right)$.

Steen et al. ${ }^{35}$, estudaram a interação do treinamento físico e a inibição da ECA na ação da insulina em ratos obesos durante 6 semanas. Os ratos tratados somente com o treinamento físico e os treinados com combinação de inibidores da ECA reduziram a hiperinsulinemia de jejum, obtiveram redução da área abaixo da curva de glicose e insulina no teste de tolerância a glicose, com aumento da ação da insulina no transporte de glicose para o interior da célula muscular.

O exercício físico promove benefícios importantes para o paciente diabético e hipertenso, desde que seja empregado na intensidade e duração adequada, respeitando o estado diabético.

\section{Exercícios Físicos Recomendados para Diabéticos Tipo 2}

Os exercícios recomendados são aqueles de característica aeróbia como caminhar, nadar, correr, andar de bicicleta, etc, que envolvem grande massa muscular, com freqüência de três a quatro vezes semanais e duração 20 a 60 minutos, não devendo ultrapassar a intensidade de $85 \%$ do $\mathrm{VO}_{2 \max }$, podendo este controle de intensidade ser feito também com base na PSE

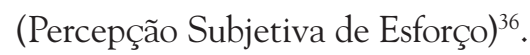

Homens japoneses diabéticos tipo 2 foram comparados com não diabéticos, durante teste incremental em cicloergômetro com análise ventilatória, no qual os pacientes diabéticos apresentaram capacidade reduzida para realizar exercício, tendo as intensidades de exercício calculadas para $60 \%$ da $\mathrm{FC}_{\text {máx }}$ e Percepção Subjetiva de Esforço (PSE) de 12, utilizandose escala Borg de 15 pontos $^{37}$, principalmente, para aqueles diabéticos com idades avançadas. Em estudo recente ${ }^{38}$, foram verificadas as intensidades de exercício correspondentes aos limiares de lactato, ventilatório e glicêmico de diabéticos tipo 2 sedentários, de indivíduos não diabéticos sedentários e também de diabéticos tipo 2 fisicamente ativos, e estas correspondiam à PSE próxima de 13 na escala de Borg de 15 pontos. De modo geral, para a maioria dos pacientes não seria recomendável a prescrição de exercícios aeróbios de intensidades superiores aos limiares (de lactato ou ventilatório), uma vez que em estudo realizado em nosso laboratório, foi observado que em exercício aeróbio realizado no domínio intenso (ex. 10\% acima do limiar anaeróbio) as respostas da pressão arterial sistólica e do duplo produto de diabéticos tipo 2 aumentavam significativamente ao longo de 
20 minutos de exercício, podendo resultar em maiores riscos cardiovasculares aos praticantes $^{39}$. Assim, apesar de termos evidenciado que a intensidade do exercício desempenha papel importante na redução da pressão arterial de DM2 pós-exercício (maior HPE na intensidade 10\% acima VS 10\% abaixo do limiar anaeróbio $)^{40}$, intensidades próximas e ligeiramente abaixo do limiar anaeróbio tem se mostrado mais seguras ${ }^{39}$ e também resultam em benefícios para o controle glicêmico e hemodinâmico de diabéticos tipo $2^{40,41}$.

A prática regular de exercícios físicos por indivíduos diabéticos, dentro das intensidades recomendadas, podem resultar em redução de $10 \%$ a $20 \%$ na hemoglobina glicosilada, e também em melhor transporte de oxigênio pela corrente sanguínea ${ }^{1}$.

Para proporcionar melhora da aptidão física, tem sido recomendada a associação entre exercícios aeróbicos e resistidos, com cargas baixas. Este tipo de associação colabora para o aumento da capacidade cardiorrespiratória, da força e resistência muscular, as quais são necessárias para uma melhor qualidade de vida, facilitando a execução de atividades da vida diária, como subir escadas, carregar compras do supermercado, etc., além de contribuir para o controle da glicemia ${ }^{11}$.

Embora existam recomendações quanto à intensidade e duração do exercício para o melhor controle do diabetes e da hipertensão, uma vez que os diabéticos geralmente desenvolvem a HAS, as relações entre o sistema renina angiotensina, calicreína-cininas para cada tipo de polimorfismo da ECA não estão bem estabelecidos.

Por meio dessa revisão, verifica-se que o exercício pode colaborar para o aumento da liberação de bradicinina, aumentando a captação da glicose durante e após realização de exercício, e também colaborar para uma redução aguda da pressão arterial pós-exercício, mesmo com a expressão aumentada de ECA em diabéticos que apresentem o alelo D.

Esse efeito agudo precisa ser investigado, averiguando o tempo de ação e o número de vezes que a bradicinina pode aumentar e ser efetiva na captação de glicose e, por conseguinte, na redução dos níveis pressóricos em diabéticos homozigotos para deleção, inserção e heterozigotos.

Estudos futuros poderiam investigar e pro- por protocolos de exercício e recuperação pósexercício específicos, que sejam sensíveis para avaliar o grau de comprometimento vascular/ endotelial (ex. ocorrência ou não de dilatação endotélio dependente e/ou de hipotensão pósexercício) e metabólico (ex. avaliação da redução da glicemia durante e após o exercício), e com isso avaliar tanto a gravidade da patologia em alguns indivíduos, e a predisposição em outros. Este tipo de avaliação tornaria ainda possível a prescrição de programas de exercício mais específicos para cada caso.

\section{CONSIDERAÇÕES FINAIS}

Com base nas evidências apresentadas por essa revisão de literatura, pôde-se concluir que o DM2 afeta de maneira efetiva os ajustes fisiológicos relacionados ao metabolismo de carboidratos, trazendo conseqüências desastrosas para os demais sistemas fisiológicos, principalmente o vascular, resultando em doenças que podem em última instância, levar à morte. O DM2 é um problema de saúde pública e pesquisas envolvendo genética, como o estudo dos polimorfismos da ECA e formas de tratamento não-farmacológico, como o exercício físico, são fundamentais para garantir futuramente a prevenção e melhorar a eficácia do tratamento do diabetes tipo 2, promovendo melhora no prognóstico dessa doença. Estudos adicionais envolvendo exercício físico, diabetes mellitus tipo 2 e polimorfismos da ECA ainda precisam ser realizados, com o intuito de analisar as respostas fisiológicas ao exercício físico e suas associações com polimorfismos genéticos específicos.

\section{REFERÊNCIAS BIBLIOGRÁFICAS}

1. American Diabetes Association. Diabetes Care 2005; 28: S37-S42.

2. Oliveira EM, Alves GB, Barauna VG. Sistema renina-angiotensina: interação gene-exercício. Rev Bras Hipertens 2003;10(2):125-129.

3. Rigat B, Hubert C, Alhenc-Gelas F, Cambien F, Corvol P, Soubrier F. An insertion/deletion polymorphism in the angiotensin I-converting enzyme gene accounting for half the variance of serum enzyme levels. J Clin Invest 1990;86(4):1343-1346.

4. Moleda P, Majkowska L, Kaliszczak R, Safranow K, Adler G, Goracy I. Insertion/deletion polymorphism of angiotensin I converting enzyme gene and left ventricular hypertrophy in patients with type 2 diabetes mellitus. Kardiol Pol 2006;64(9):959-965. 
5. Bhoola KD, Figueroa CD, Worthy K. Bioregulation of kinins: Kallikreins, kininogens, and kininases. Pharmacol Rev 1992;44(1):1-80.

6. Shiuchi T, Cui TX, Wu L, Nakagami H, Takeda-Matsubara Y, Iwai M etal. ACE inhibitor improves insulin resistance in diabetic mouse via bradykinin and NO. Hypertension 2002;40(3):329-334.

7. Arzu Ergen H, Hatemi H, Agachan B, Camlica $\mathrm{H}$, Isbir T. Angiotensin-I converting enzyme gene polymorphism in Turkish type 2 diabetic. Exp Mol Med 2004;36(4):345-350.

8. Larsen JJ, Dela F, Madsbad S, Galbo H. The effects of intense exercise on postprandial glucose homeostasis in type II diabetic patients. Diabetologia 1999;42(11):1282-1292.

9. Poirier P, Mawhinney S, Grondin L, Tremblay A, Broderick T, Cléroux J, et al. Prior meal enhances the plasma glucose lowering effect of exercise in type 2 diabetes. Med Sci Sports Exerc 2001;33(8):1259-1264.

10. Silva CA, Lima WC. Efeito benéfico do exercício físico no controle metabólico do diabetes mellitus tipo 2 à curto prazo. Arq Bras Endocrinol Metab 2002;46(5):550-556.

11. Tokmakidis SP, Zois CE, Volaklis KA, Kotsa K, Touvra AM. The effects of a combined strength and aerobic exercise program on glucose control and insulin action in women with type 2 diabetes. Eur J Appl Physiol 2004;92(4-5):437-442.

12. Combettes-Souverain M, Issad T. Molecular basis of insulin action. Diabetes Metab 1998; 24(6):477-489.

13. Shulman GI. Cellular mechanisms of insulin resistance. J Clin Invest 2000;106(2):171-176.

14. El-Atat FA, Stas SN, Mcfarlane SI, Sowers J. The relationship between hyperinsulinemia, hypertension and progressive renal disease. J Am Soc Nephrol 2004;15(11):2816-2827.

15. Wild S, Roglic G, Green A, Sicree R, King H. Global prevalence of diabetes: estimates for the year 2000 and projections for 2030. Diabetes Care 2004;27(5):1047-1053.

16. Consenso Brasileiro sobre Diabetes. Diagnóstico e classificação do diabetes melito e tratamento do diabetes melito tipo 2. Sociedade Brasileira de Diabetes. Rio de Janeiro: Diagraphic; 2002.

17. Cavalheira JBC, Zecchin HG, Saad MJA. Vias de Sinalização da Insulina. Arq Bras Endocrinol Metab 2002;46(4):419-425.

18. Savage DB, Petersen KF, Shulman GI. Mechanisms of insulin resistance in humans and possible links with inflammation. Hypertension 2005;45(5):828-833.

19. Groop PH, Forsblom C, Thomas MC. Mechanisms of disease: pathway-selective insulin resistance and microvascular complications of diabetes. Nature Clin Pract 2005;1(2):100-110.

20. Fleming I, Kohlstedt K, Busse R. The tissue renin angiotensin system and intracellular signaling. Current Opin Nephrol Hypertens 2006;15(1):8-13.
21. Wang Y, Magi CY, So WY, Tong PCY, Ronald CW, Chow CC, et al. Prognostic effect of Insertion/Deletion polymorphism of the ACE gene on renal and cardiovascular clinical outcomes in Chinese patients with type 2 diabetes. Diabetes Care 2005;28(2):348-354.

22. Panahloo A, Andres C, Mohamed-Ali V, Gould MM, Talmud P, Humphries SE, Yudkin JS. The insertion allele of the ACE gene I/D polymorphism. A candidate gene for insulin resistance? Circulation 1995;92(12):3390-3393.

23. Molnar GA, Wagner Z, Wagner L, Melegh B, Koszegi T, Degrell P, et al. Effect of ACE carbohydrate metabolism, on oxidative on end-organ damage in type-2 diabetes mellitus. Orv Hetil 2004;145(16):855-859.

24. Lee YJ, Tsai, JCR. ACE gene insertion/deletion polymorphism associated with 1998 world health organization definition of metabolic syndrome in Chinese type 2 diabetic patients. Diabetes Care 2002;25(6):1002-1008.

25. Costa AC, Canani LH, Maia AL, Gross JL. The ACE Insertion/Deletion polymorphism is not associated with the metabolic syndrome (WHO definition) in Brazilian type 2 diabetic patients. Diabetes Care 2002;25(12):2363-2364.

26. So WY, Ma RC, Ozaki R, Tong PC, Ng MC, Ho $\mathrm{CS}$, et al. Angiotensin-converting enzyme (ACE) inhibition in type 2, diabetic patients - interaction with ACE insertion/deletion polymorphism. Kidney Intern 2006; 69(8):1438-1443.

27. Taguchi T, Kishikawa H, Motoshima H, Sakai K, Nishiyama T, Yoshizato K, et al. Involvement of bradykinin in acute exercise-induced increase of glucose uptake and GLUT-4 translocation in skeletal muscle: studies in normal and diabetic humans and rats. Metabolism 2000;49(7):920-930.

28. Henriksen SJ, Kinnick EB, Youngblood EB, Schmit MB, Dietze GJ. Ace inhibition and glucose transport in insulin-resistant muscle: roles of bradykinin and nitric oxide. Am J Physiol Regulatory Integrative Comp Physiol 1999;277(1): R332-R336.

29. Forjaz CL, Matsudaira Y, Rodrigues FB, Nunes N, Negrão CE. Post-exercise changes in blood pressure, heart rate and rate pressure product at different exercise intensities in normotensives humans. Braz J Med Biol Res 1998;31(10):1247-1255.

30. Brandão Rondon MU, Alves MJNN, Braga AMFW, Teixeira OTUN, Barreto ACP, Kreiger EM, et al. Postexercise blood pressure reduction in elderly hypertensive patients. J Am Coll Cardiol 2002;39(4):676-682.

31. Halliwill JR. Mechanisms and clinical implications of postexercise hypotension in humans. Exerc Sport Sci Rev 2001;29(2):65-70.

32. MacDonald JR, Hogben CD, Tarnopolsky MA, MacDougall JD. Post exercise hypotension is sustained during subsequent bouts of mild exercise 
and simulated activities of daily living. J Hum Hypertens 2001;8(15):567-71.

33. Moraes MR, Bacurau RF, Ramalho JD, Reis FC, Casarini DE, Chagas JR et al. Increase in kinins on post-exercise hypotension in normotensive and hypertensive volunteers. Biol Chem 2007;388(5):533-540.

34. Pontes Jr FL, Bacurau RF, Moraes MR, Navarro F, Casarini DE et al. Kallikrein kinin system activation in post-exercise hypotension in water running of hypertensive volunteers.Int Immunopharmacol 2008; 8(2):261-266.

35. Steen MS, Foianini EB, Youngblood EB, Kinnick TR, Jacob S, Henriksen EJ. Interactions of exercise training and ACE inhibition on insulin action in obese Zucker rats. J Appl Physiol 1999;86(6):20442051.

36. American College of Sports Medicine. Manual de pesquisa das diretrizes do ACSM para os testes de esforço e sua prescrição. Rio de Janeiro: Guanabara Koogan; 2003.

37. Kunitomi M, Takahashi K, Wada J, Suzuki H, Miyatake N, Ogawa S, et al. Re-evaluation of exercise prescription for Japanese type 2 diabetic patients by ventilatory threshold. Diabetes Res Clin Pract 2000;50(2):109-115.

38. Moreira SR, Simões GC, Hiyane WC Campbell, CSG, Simões, HG. Identificação do limiar anaeróbio em indivíduos com diabetes tipo-2 sedentários e fisicamente ativos. Rev Bras Fisioter 2007;(11):289-296.
39. Do Valle G, Simões HG, Hyane W, Moreira SR, Ohata I, Campbell CSG. Respostas cardiovasculares de diabéticos tipo 2 durante exercício realizado em diferentes intensidades. Diabetes Clínica 2006;(10):271-276.

40. Lima LC, Assis GV, Hiyane W, Almeida WS, Arsa G, Baldissera V, Campbell CSG, Simões HG. Hypotensive effects of exercise performed around anaerobic threshold in type 2 diabetic patients. Diabetes Res Clin Pract 2008;81(2): 216-22.

41. Hiyane W, Moreira SR, Ferreira CES, de Souza MV, de Oliveira RJ, Campbell CSG, Simões HG. Blood glucose responses of type-2 diabetics during and after exercise performed at intensities above and below anaerobic threshold. Revista Brasileira de Cineantropometria \& Desempenho Humano 2008; 1(10): 8-11.

\footnotetext{
Endereço para correspondência

Gisela Arsa da Cunha

Endereço: Rua Prefeito José de Melo Franco, n. 136 - Jardim Universo CEP 08740-540 - Mogi das Cruzes - SP

E-mail: gisarsa@gmail.com
} 